\title{
Quantum effects in tunnelling plasmonics
}

\author{
J. Aizpurua ${ }^{1}$, R. Esteban ${ }^{1}$, P. Nordlander ${ }^{2}$, A. Borisov $^{3}$ \\ 1. Materials Physics Center (CSIC-UPV/EHU) and Donostia International Physics Center DIPC, Donostia-San Sebastián, Spain \\ 2. Department of Physics, Laboratory for Nanophotonics, Rice University, Houston, Texas, USA \\ 3. Institut des Sciences Moléculaires d'Orsay-UMR 8214, CNRS- Université Paris-Sud, Orsay, France
}

The optical response of metallic nanoparticles shows antenna resonance effects at optical frequencies derived from the collective excitation of the conduction electrons at the boundaries of the particles, so called surface plasmons. This response is commonly described in the framework of classical electrodynamics by means of a linear polarizability obtained within a local or nonlocal approach of the excitations. Alternatively, quantum mechanics within time-dependent density functional theory (TDDFT) offers an appropriate framework to fully address the complex exchange and correlations of the electron gas in the metal together with an accurate description of the geometrical boundaries of the particles through the corresponding potential barriers. In Fig. 1 we show the differences between a classical and a quantum treatment of the optical response for the two lowest bonding plasmon modes in a Na nanodimer. Quantum effects derived from the spill out of the electrons at the boundaries and from the electron tunnelling between particles are properly addressed within the TDDFT showing clear differences with respect to a classical treatment. However the number of electrons that can be considered in the quantum calculation is typically limited to a few thousands of electrons, therefore far from realistic plasmonic systems that usually involve millions or billions of electrons. Here we introduce a quantumcorrected model (QCM) that integrates the quantum response of a metallic cavity with the macroscopic response of the rest of the plasmonic system [1]. This hybrid response accounts properly for quantum effects derived from the coherent tunnelling across the cavity while tracing the macroscopic plasmonic modes.
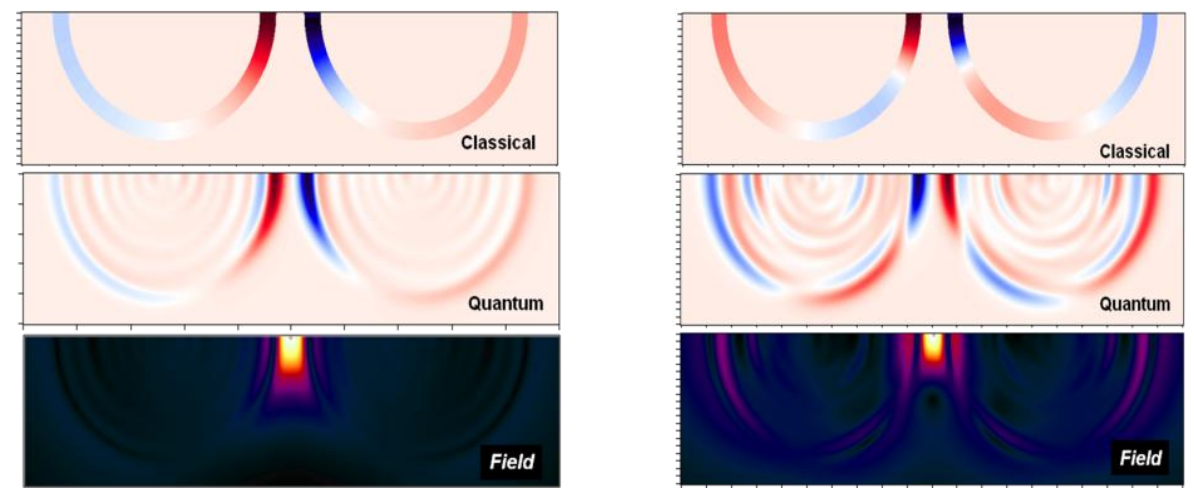

Fig. 1 Surface charge density induced in a Na dimer of $2 \mathrm{~nm}$ particles separated by $6 \AA$ calculated within a classical formalism (top) and a quantum formalism (middle). The corresponding near-field maps are displayed at the bottom. The lowest energy bonding dimer Plasmon (BDP) at $\hbar \omega=2.55 \mathrm{eV}$ is shown to the left whereas the quadrupolar bonding plasmon (QBP) at $\hbar \omega=3.24 \mathrm{eV}$ is shown to the right.

We apply the quantum-corrected model (QCM) to interpret experimental results in plasmonic cavities where two metallic nanoparticles are located in subnanometric proximity [2]. As the particles reach a few Angstroms of separation, quantum tunnelling between the particles is triggered out and the bonding dimer plasmon (BDP) gets screened and blue-shifts until it disappears from the spectrum. Due to the tunnelling, new charge-transfer modes emerge even before physical contact of the nanoparticles. The chromatic fingerprint of the quantum regime is clearly revealed both theoretically and experimentally and the results can be interpreted within the quantum-corrected model of the plasmonic response. Furthermore, TDDFT can address nonlinear effects derived from quantum tunnelling that produce an even more pronounced effect in the screening of the near-field inside the cavity, further reducing the fieldenhancement in it [3]. As the control of the sizes of the structures reaches subnanometric scale, quantum effects become of paramount importance in the understanding of the optical response of plasmonic systems, opening the pathway to exotic nonlinear effects within ultrafast coherent control in nanooptics.

\section{References}

[1] R. Esteban, P. Nordlander, A.G. Borissov and J. Aizpurua, "Bridging quantum and classical plasmonics with a quantum-corrected model," Nat. Communications. 3, 825 (2012).

[2] K.J. Savage, M.M. Hawkeye, R. Esteban, A.G. Borisov, J. Aizpurua and J.J. Baumberg, "Revealing the quantum regime in tunnelling plasmonics," Nature 491, 574 (2012).

[3] D.C. Marinica, A.K. Kazansky, P. Nordlander, J. Aizpurua and A.G. Borissov, "Quantum plasmonics: nonlinear effects in the field enhancement of a plasmonic nanoparticle dimer," Nano. Lett. 12, 1333 (2012). 